\title{
Effects of Nitrate and Pathogenic Nanoparticles on Reproductive Losses, Congenital Hypothyroidism and Musculoskeletal Abnormalities in Mares and Other Livestock: New Hypotheses
}

\author{
Thomas Walter Swerczek*, Alan Ray Dorton \\ Department of Veterinary Science, University of Kentucky, Lexington, U.S
}

Email address:

thomas.swerczek@uky.edu (T. W. Swerczek)

${ }^{*}$ Corresponding author

\section{To cite this article:}

Swerczek Thomas Walter, Dorton Alan Ray. Effects of Nitrate and Pathogenic Nanoparticles on Reproductive Losses, Congenital Hypothyroidism and Musculoskeletal Abnormalities in Mares and Other Livestock: New Hypotheses. Animal and Veterinary Sciences. Vol. 7, No. 1, 2019, pp. 1-11. doi: 10.11648/j.avs.20190701.11

Received: December 20, 2018; Accepted: January 20, 2019; Published: February 13, 2019

\begin{abstract}
Spontaneous abortions, congenital hypothyroidism and musculoskeletal abnormalities were attributed to high nitrate in the diet of pregnant mares on Thoroughbred farms in central Kentucky. These fetal losses, with an unknown etiology, and associated with the Mare Reproductive Loss Syndrome (MRLS) have plagued horse farms in central Kentucky for decades. Fetal losses occur in mares grazing spring pastures affected by climatic and environmental factors including droughts, cold-stress, nitrogenous fertilizers, and herbicides. These factors may cause nitrate to accumulate in pasture forages. On a Thoroughbred horse farm, mares affected with the MRLS, pregnant Boer goats grazing high nitrate pastures also were affected with fetal losses. When spring pastures were not fertilized with nitrogen, herbicides not applied, protein reduced in the ration and the diet increased in sodium, fetal losses did not occur and foals were normal at birth. Excessive nitrate, ammonia and sulfate in the diet were associated with the formation of toxic and pathogenic abiotic nanoparticles in the amnionic fluid and pathognomonic placental lesions consistent with the MRLS. Pathogenic nanoparticles were found in aborted fetuses of other livestock. The discovery of these toxic pathogenic abiotic micro and nanoparticles in developing fetuses is unique. This novel mechanism of action for the pathogenesis of fetal losses may be a predisposing factor for a host of opportunistic diseases in livestock. The pathogenic nanoparticles collect in the vessels of the placenta and other organs to form niduses that predispose fetuses to a host of opportunistic microorganisms.
\end{abstract}

Keywords: Nitrate Toxicity, Pathogenic Nanoparticles, Fetal Loss, Congenital Hypothyroidism, Musculoskeletal Abnormalities

\section{Introduction}

During the average foaling season in central Kentucky 5 to 15 percent early fetal deaths occur in mares. Most fetuses are not found for examination. An additional 2 to 5 percent of mid to late-term abortions are found for necropsy examination [1]. Most fetal losses have an unknown etiology and are related to drastic environmental and climatic changes that affect pasture forages in the late spring and to a lesser extent in the early fall. During some foaling seasons, these reproductive losses may dramatically increase in late spring.
An extraordinary increase in abortions was first recognized as a syndrome in the late spring of 1980 after pasture forages were stressed by droughts, frosts and freezes, and affected pasture forages and diets were found to be elevated in nitrate [2] Since 1980, similar fetal losses were recognized each spring. However, in the spring of 2001, there was another massive unprecedented increase in fetal losses when several thousand mares acutely aborted in central Kentucky and Midwestern states [3]. Fetal losses were referred to as the Mare Reproductive Loss Syndrome (MRLS) [4] However, the fetal loss syndrome was not only restricted to mares but 
to other livestock [5] Like in 1980, late spring pastures were unseasonably stressed by frosts and freezes. Cold-stress to pasture forages will induce an acute pike in potassium and nitrate in damaged plants. As in 1980, high potassium and nitrate were found in affected cold-stress pasture forages in 2001. In addition to nitrate toxicosis, ammonia toxicosis, as a product of excessive nitrate, was a common finding and a suggested contributing etiologic factor for the MRLS [6]. Also, saprotrophic and commensal bacteria that infect frost damaged pastures were found to contribute to gut microbial overgrowth and lesions associated with the MRLS [7].

After unseasonable late spring frost and freeze damage to high nitrogenous pasture forages, followed by an extreme weather swing to high climatic temperatures, decaying forages will ferment and take on a haylage-like and ammonia smell. Microorganisms in decaying pasture forages are likely associated with loose odoriferous feces pregnant mares that abort frequently experience before fetal losses [7]. In addition to loose feces, some mares may experience mild signs of laminitis. There are no changes in the reproductive hormone values in mares that abort. Clinical pathologic laboratory tests are negative with the exception of elevated nitrate and ammonia in the blood of mares [6].

Increased reproductive losses in 1980 and 2001 were most numerous in early developing fetuses, but they occurred at all stages of gestation starting 9 to 10 days after pasture forages were severely stressed [5]. Nitrate increases in pasture forages within 24 to 48 hours after cold-stress, and will remain elevated until the plant recovers with new growth. The syndrome is more common after unseasonable late spring erratic weather patterns of droughts and cold-stress to pasture forages.

Nitrate has a relaxing effect on the vascular smooth muscles and apparently initiates the remarkable dilation of the cervix that occurs prior to and during the fetal losses. The first clinical sign of impending fetal loss in early developing embryos and fetuses is heart failure, followed by abortion in a few days. If the early developing embryos and fetuses survive acute death, later in development, the vessels of the amnion become hyperplasic, tortuous and beaded in appearance with moderate to marked perivascular edema and focal hemorrhage. The amnionic umbilical cord becomes enlarged and may contain a fibrinous inflammation on the serosa or an apparent funititis. The allantochorion may be thickened due to edema. The edema in the amnion and allantochorion increases the weight of the placenta from a normal average of 10 to 12 pounds, to 18 to 20 pounds in some affected cases.

These gross pathologic lesions in the placenta are pathognomonic. In opportunistic free infections, which are rare, microscopic lesions are primarily vascular hyperplasia and perivascualar edema in the amnion and amnionic umbilical cord. In some fetuses, the amnion is infarcted in patchy areas and stained with meconium due to a fetal diarrhea. Circular calcified plaques, 1 to $2 \mathrm{~cm}$ in diameter, may be present in the amnion. Cysts and constrictions often develop in the amnionic umbilical cord and may cause an obstruction to the circulation in the fetus. The lesions in the umbilical cord are clearly congenital developmental anomalies as are the musculoskeletal abnormalities, primarily limb contractures that are associated with the fetal loss syndrome.

With affected fetuses that do not abort early in gestation, congenital abnormalities may occur in the umbilical cord and the musculoskeletal system, primarily limb contractures. The severe and fatal congenital anomalies represent a small percentage of the fetal losses. However, minor limb contractures, umbilical cord and amnion lesions are more common and often not fatal. Musculoskeletal abnormalities account for significant losses in newborn foals and mares may die during parturition due to dystocia and foaling complications related to limb contractures. Developmental anomalies, including the contracted foal syndrome, with an unknown etiology have been affecting foals in central Kentucky $[8,9]$. and elsewhere for decades. Musculoskeletal anomalies often occur in association with placental changes (10).

In a 13-year survey between 1970 and 1982, 608 deformed foals were examined at necropsy, and 202 newborn foals that had musculoskeletal abnormalities, primarily fore and hind limb contractures. In addition to the limb contractures, scoliosis and torticollis were also occasionally present. Some foals with the contracted limbs had cranial asymmetry or curvature, with a deviated premaxilla (wry nose). Rarely, malformations of occipital and atlas bones at their articulation were present. The contractures of the fore and hind limbs involved all joints to various degrees, but the metacarpophalangeal and metatarsophalangeal joints were most frequently affected. The foals with the contracted foal syndrome were born at or near term. There did not appear to be a breed prevalence. Since the thyroid gland of foals was not grossly enlarged, it was not routinely examined histomorphologically [9].

Since the mysterious fetal losses were first recognized as a definitive syndrome during the 1980 breeding season [2] we have observed several thousand reproductive loss cases clinically and at postmortem. These fetal losses, with mysterious etiologies, have plagued the breeding industry for numerous decades. The following natural spontaneous cases are representative of the many that occur on breeding operations involving mares and other herbivores.

\section{Materials and Methods}

\subsection{Effects of Nitrate Toxicosis on Pregnant Boer Goats}

A herd of twenty pregnant Boer goats were housed together and grazed on a pasture of bluegrass, orchard grass and white clover. During the 2015 kidding season, while the pregnant goats were in the first trimester, the pasture was fertilized with 100 pounds of ammonium nitrate per acre. Three does aborted within the first 30 days after the pasture was fertilized with ammonium nitrate. The placental membranes were edematous and congested, and the fetuses 
were free of other lesions. Six does delivered premature kids, which were born hairless with skin edema. All premature kids had a very enlarged thyroid gland. The fore and hind legs in one kid goat were contracted at the metacarpal and metatarsal phalangeal joints. The placentas of the kid goats were edematous and congested.

Microscopic examination of $\mathrm{H}$ and $\mathrm{E}$ stained histologic sections of the thyroid gland from affected kid goats revealed marked thyroid follicular hyperplasia. The follicles throughout the thyroid were small, angular and dense with hypercellular cuboidal and columnar epithelium. Very little colloid was present in the follicles.

Eleven does delivered kids at term with all having mild to moderate goiter or thyroid hyperplasia. Three kids had contractures of the fore, and hind limbs at the metacarpal and metatarsal phalangeal joints. All three developed periodic seizures and died 5 to 7 days postpartum. Eight does delivered kid goats that had mild thyroid hyperplasia with a visible thyroid gland at birth, but the thyroid gland gradually reduced in size at three months of age and was no longer palpable.

\subsection{Effects of Nitrate Toxicosis on Pregnant Mares and Boer Goats}

On Thoroughbred horse farm A, pregnant mares and pregnant Boer goats were grazing on suspected high nitrate spring pastures. The pastures where the mares and goats were grazing consisted of bluegrass, orchard grass, fescue and abundant white clover. The supplemental hay was primarily high protein alfalfa. Pastures were treated with a herbicide (a) in the early spring. Later during the spring, frosts and freezes damaged the pasture forages, and six mares in the third trimester of gestation aborted. The pathologic lesions in all aborted fetuses were consistent with the MRLS [4]. The amnion and amnionic umbilical cord in all foals were edematous and the hyperplastic vessels contained focal areas of hemorrhage. Allantochorion was thickened due to edema. No other significant gross lesions were present in other fetal organs. Pregnant Boer experienced fetal losses, thyroid hyperplasia and musculoskeletal abnormalities. The pathologic lesions in the kid goats were identical to the affected kid goats from the above Boer goat herd grazing pastures fertilized with ammonium nitrate.

\subsection{Effects of Nitrate Toxicosis on a Herd of Pregnant Mares}

Thoroughbred-breeding farm B had experienced fetal losses consistent with the MRLS each year for the previous 10 years or more. Mares on the farm had low conception rates, early and late abortions, and foals were born with musculoskeletal abnormalities. The history, clinical signs and congenital defects that primarily affected the fore and hind legs were identical to those that had been seen on many other farms in central Kentucky for several years [9].

Pastures where the pregnant mares were grazing consisted of bluegrass, orchard grass, perennial ryegrass, fescue and white clover. The pastures were infested with a variety of weeds, including creeping yellow buttercups throughout the pastures, and ragweeds and pigweeds in heavily grazed areas. Pastures were fertilized each spring with 100 pounds of urea per acre as a nitrogen source and treated with a herbicide (a). Forage during winter months was primarily legume-grass hay, with approximately $50 \%$ fescue grass. The supplemental grain ration consisted of a $14 \%$ protein sweet feed, which was a mixture of corn, oats, soybean meal, molasses and was fortified with minerals, vitamins and $0.5 \%$ salt.

During the 2014 foaling season, 12 mares were bred, six mares lost their pregnancies early in gestation, and six mares foaled near term. Only four the foals were normal at birth, and two foals were born with musculoskeletal abnormalities. For several previous foaling seasons, similar reproductive losses of unknown etiologies occurred.

Two foals were born in the spring of 2015 with congenital musculoskeletal defects. The first foal with congenital defects was a filly born on 5-12-15 after a breeding date of 65-14. The dam of the foal did not develop an udder before foaling. The premaxilla bone was severely deviated forming a wry nose. The flexor tendons in the forelegs were contracted. Part of the small intestine was eviscerated through an incomplete closure of the ventral abdominal wall near the navel. The synovial membranes in the limb joints were congested and edematous. The fore and hind legs were radiographed at necropsy and there appeared to be normal ossification of the carpal and tarsal bones. The thyroid was not enlarged. The placenta was not submitted for examination. Other organs were grossly normal.

Histopathologic examination of $\mathrm{H}$ and $\mathrm{E}$ stains of the thyroid gland revealed that the follicles were smaller than normal with less colloid than normal. These follicles were lined with large cuboidal epithelial cells. In focal areas the follicles were dilated, larger than normal with abundant colloid, and lined with small cuboidal epithelial cells.

One day later, a colt was born on 5-13-15 after a breeding date of 6-8-14. The dam of the foal prematurely developed an udder before foaling, which indicated a premature foaling was imminent. The skull was malformed with a domed forehead. The premaxilla bone was deviated laterally. Eye sockets were off set and bilaterally asymmetrical. Occipital and atlas bones were malformed and the skull was deviated laterally. The flexor tendons in the fore and hind legs were contracted. Fore and hind legs were radiographed at necropsy, and there was normal ossification of the carpal and tarsal bones.

The allantochorion appeared normal, but the amnionic vessels were hyperplasic, tortuous and beaded in appearance and very edematous with focal areas of hemorrhage.

(a). Crossbow contains 2,4-dichlorophenoxyacetic acid, butoxyethyl ester, Triclopyr BEE.

The amnionic umbilical cord was hyperplasic, edematous and hemorrhagic. Thyroid gland was not enlarged. There were no gross lesions in other organs. Histopathologic examination of $\mathrm{H}$ and $\mathrm{E}$ stains of the thyroid gland revealed that the follicles were smaller than normal with less colloid 
than normal. These follicles were lined with large cuboidal epithelial cells and some columnar epithelial cells. The smaller follicles were hypercellular with very little colloid. In other areas, the follicles were normal in morphology and in the amount of colloid.

\subsubsection{Treatment to Lessen Dietary Nitrate in Pasture Forages for a Herd of Pregnant Boer Goats}

In the pasture where the Boer goat herd was housed, no nitrogenous fertilizers or herbicides were applied to the pasture during the 2016 kidding season.

\subsubsection{Treatment to Eliminate Dietary Nitrate from Pasture Forages for Pregnant Mares}

After the sixth mare aborted on Thoroughbred farm A, all remaining pregnant mares in the herd were removed from the high nitrate pasture forages and moved to a dry lot and fed mixed grass-legume hay and a supplemental grain ration. The pregnant Boer goats were not removed from the pastures where the mares aborted.

\subsubsection{Treatment to Reduce Dietary Nitrate from Pasture Forages and Feed Rations for Pregnant Mares}

Since the Thoroughbred horse farm B experienced the same or similar reproductive losses over approximately tenyear period, it was suspected that the poor reproductive performance in the mares was associated with excessive nitrogenous compounds in the diet from pasture forages and the grain supplement. During the 2016 foaling season, it was recommended that pastures should not be fertilized or treated with herbicides. The grain supplement was reduced to a $12 \%$ protein sweet feed, and natural mineralized salt increased from $0.5 \%$ to $1.5 \%$ of the total feed ration. The supplemental hay was recommended to be primarily a non-legume grass.

\section{Results}

\subsection{Results After Dietary Nitrates Were Reduced in Pasture Forages for Pregnant Boer Goats}

During the 2016 kidding season, after no nitrogenous fertilizers were applied to the pasture, there were no fetal losses or goiter in any of the newborn kid goats. In the Boer goat herd during the previous several kidding seasons, there were no cases of goiter in the kid goats. Goiter in the newborn kid goats only occurred when the ammonium nitrate was added to the pasture while the pregnant does were grazing during the 2015 kidding season as described in section 2.1 .

\subsection{Results After Dietary Nitrates Were Reduced for Pregnant Mares, and not for Pregnant Boer Goats}

On Thoroughbred horse farm A, after the pregnant mares were transferred to a dry lot, all mares delivered normal foals at term. However, the pregnant does that remained in the herbicide treated and cold-stressed pasture forages, $40 \%$ experienced fetal losses, including marked congenital thyroid hyperplasia and musculoskeletal abnormalities of the limbs.
The gross and microscopic lesions in the affected kid goats were identical to those seen in kid goats from pregnant does grazing ammonium nitrate-fertilized pastures as described in section 2.1 .

\subsection{Results After Dietary Nitrates Were Reduced in the Diet for a Herd of Pregnant Mares}

On the Thoroughbred farm B, twelve mares were bred during the 2016 foaling season, and eleven mares conceived. Ten healthy foals were born with no musculoskeletal abnormalities. One mare aborted normal twin fetuses at midgestation. After changing the diet fed to pregnant mares and pasture management to reduce the likelihood of excessive nitrate in pasture forages, for the first time in over ten years, all foals were born without musculoskeletal abnormalities, and no dystocia occurred in any of the mares. Six mares were re-bred during the 2017 foaling season and all had normal foals in 2018 .

\section{Discovery of Natural Occurring Micro and Nanoparticles}

\subsection{Natural Occurring Fetal Losses Associated with the MRLS}

Our epidemiological evidence suggests that fetal losses associated with the MRLS are consistent with nitrate toxicosis that induces a variety of pathologic lesions. Paradoxically, pathognomonic lesions in the amnion and amnionic umbilical cord suggest that a specific novel pathogenic uncultivatable entity may be the causative etiologic agent. However, only a host of commensal and nonpathogenic bacteria common in soil and pasture, mouth and gastro-intestinal tract like non-beta hemolytic slow-growing Streptococcus spp. are present and can be cultured [[7]. A similar syndrome to the MRLS, that has been diagnosed in Australia and referred to as Equine Amnionitis and Fetal Loss (EAFL), is also associated with a host of commensal and non-pathogenic bacteria [11]. Our collaborative evaluations of affected fetal losses with workers in Australia have revealed the syndromes in both areas are identical. They feel the syndrome in Australia may have been present in lesser numbers and not diagnosed years earlier.

\subsection{Discovery of Pathogenic Nanoparticles in Fetal Loss Specimens}

After exhaustive attempts since the 1980 breeding season in central Kentucky to identify a causative agent, we have failed to isolate any significant primary pathogenic microorganisms. However an apparent uncultivable entity could be demonstrated in the amnionic fluid of affected fetuses from mares and other livestock. With the aid of transmission and scanning electron microscopic studies, micro and nanoparticles were discovered in the amnionic fluid, amnion and amnionic umbilical cord in the edematous perivascular fluid of affected organs. This discovery explains 
the puzzling phenomenon as the suspected uncultivatable entity proved to be abiotic micro and nanoparticles that can mimic pathogenic infectious microorganisms on how they induce tissue reactions and disease syndromes.

Consistent clinical findings in fetuses affected with the MRLS are aggregates of echogenic particles in the amnionic fluid. These aggregates in the amnionic fluid form a few days before fetal losses occur in early developing embryos or in fetuses due to heart failure. Their morphology can be imaged with Scanning Electron Microscopy (Figure 1). Analysis of the nanoparticles with Focused Ion Beam Scanning Electron Microscopy revealed mineral content of primarily calcium among other lesser minerals. Special stains of the micro and nanoparticles were positive for proteinaceous content. The micro and nanoparticles were negative for DNA.

With the aid of scanning electron microscopic studies of affected amnionic fluid, in addition to the micro and nanoparticles, fibrinous filaments resembling fungal hyphae with abiotic micro and nanoparticles attached to the filaments were also demonstrated. This finding may explain the presence of apparent funisitis often present on the serosa of the amnionic umbilical cord.

After the recent discovery of the abiotic nanoparticles, it was suspected that the formation of the nanoparticles in affected fetuses was associated with nitrate toxicosis.

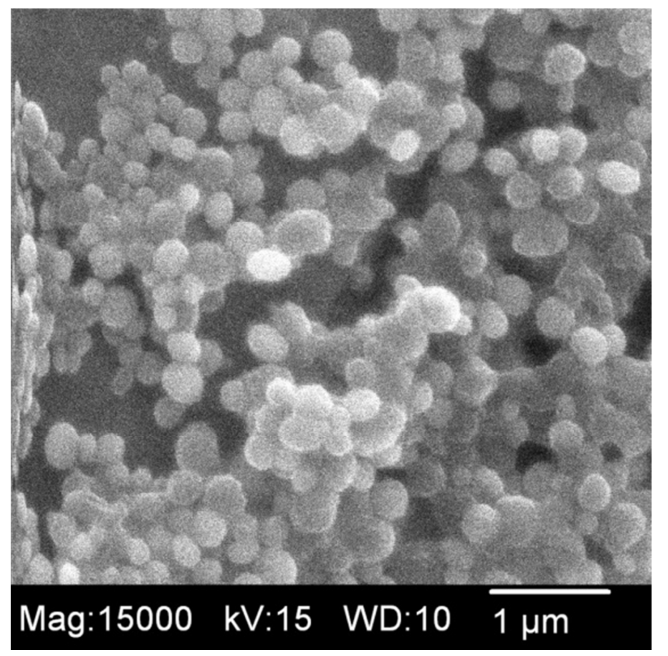

Figure 1. Scanning electron microscopic image of micro and nanoparticles in the amnionic fluid of an equine fetus with lesions of the Mare Reproductive Loss Syndrome. 15,000 X.

In addition to nitrate, excessive ammonia [6] as a metabolic by-product of nitrate, and sulfate [12] in the environment appear to be associated with the formation of the seemingly toxic and pathogenic nanoparticles. Our epidemiological studies have revealed that the fetal loss syndrome is endemic on farms with high nitrate pasture forages and concurrent high sulfur drinking water for affected pregnant mares.

With this epidemiologic hint, it was hypothesized that sulfur may be the previously unidentified factor responsible for the production of the micro and nanoparticles. This observation is consistent with recent findings by other international workers [12].

\subsection{Experimental Production of Nanoparticles in Vitro}

Since these unexplained mysterious fetal losses are associated with erratic climatic changes, we hypothesized that the micro and nanoparticles present in the amnionic fluid and placental lesions may be induced by common elements including nitrate, ammonia and sulfur that are associated with air pollution. To test this novel hypothesis these elements were experimented in vitro. Identical morphologic micro and nanoparticles were produced in vitro by adding ammonium sulfate to normal equine amnionic fluid. In addition, when other sulfonated minerals, including potassium sulfate, ferrous sulfate, magnesium sulfate, zinc sulfate and calcium sulfate were added to the same normal equine amnionic fluid, similar morphologic nanoparticles in lesser amounts were also produced. However, when the same minerals in the oxide and carbonate forms were added to the same normal equine amnionic fluid, no micro and nanoparticles were produced.

When sulfonated minerals were added to a solution of normal chicken egg yolks, similar micro and nanoparticles were produced. In natural spontaneous cases of previously unexplained cases of embryonic and fetal loss in embryonating chicken embryos, we found early embryonic and fetal losses with vascular lesions similar to those seen in equine placentas affected with amnionitis. The yolk of naturally affected embryonating chicken embryos contained huge numbers of micro and nanoparticles similar in morphology to those found in amnionic fluid of affected equine fetuses. In chicken fetuses that did not die, often chicks would not hatch or they were weak at hatching. The yolk sac was often retained and not completely absorbed. The pathogenic nanoparticles associated with the fetal loss syndrome in chickens may be currently contributing to lower than expected hatching rates in chickens and other poultry. Ammonia and sulfate in the environment are apparently involved with the induction of toxic nanoparticles (12). The egg yolk of chicken eggs is high in sulfur and ammonia is a common toxic factor in poultry operations. In addition to equine and chicken fetuses, identical pathogenic micro and nanoparticles were found in aborted fetuses of other species of livestock including caprine, bovine and porcine fetuses with unknown etiologic agents. Similar nanoparticles were also found in honey bees affected with the colony collapse disorder.

\subsection{Experimental Production of Nanoparticles in Vivo in Early Developing Chicken Embryos}

To test the effect of sulfonated minerals have in vivo, seven-day-old chicken embryos were inoculated in ovo into the yolk sac with a $1 \mathrm{cc}$ sterile water solution of $0.25 \%$. concentration of various sulfonated minerals that are commonly added to livestock rations. The sulfonated minerals tested in vitro in amnionic fluid and in vivo in developing chicken embryos were ammonium sulfate, 
potassium sulfate, ferrous sulfate, magnesium sulfate, zinc sulfate, copper sulfate, and calcium sulfate.

\subsection{Results After Sulfonated Minerals Were Inoculated into Early Developing Chicken Embryos}

Focal vascular hemorrhagic lesions were induced in the vessels of the yolk sac within 4 to 5 days after inoculation with all sulfonated minerals tested. Fetal deaths with vascular lesions consistent with those found in the amnion and amnionic umbilical cord of fetal losses of equine abortions were induced in the yolk sac of embryonating chicken eggs. A huge number of micro and nanoparticles were induced in the yolk of embryonating eggs and resulted in a high incidence of fetal deaths. The pathologic lesions in the experimental fetal losses were identical to the lesions occurring naturally in chickens with an unknown etiology. There was no evidence of any microorganisms in the inoculated fertile eggs that experienced fetal loss.

\section{Discussion}

On Thoroughbred horse farm A where mares were aborting due to suspected nitrate toxicosis, a herd of pregnant Boer goats and pregnant mares were grazing similar pasture forages. The kid goats were born with marked congenital goiter. The gross and microscopic lesions in the affected kid goats were identical to those seen in kid goats from pregnant does grazing ammonium nitrate-fertilized pastures. None of the adult goats on either farm were affected with clinical signs of goiter. Over the previous several kidding seasons and entire history of the Boer goat herd there were no cases of goiter in any newborn kid goats. During this time no nitrogenous fertilizers were added to pastures. Seemingly, the pregnant Boer goat, and possibly other small ruminants, are ideal sentinel herbivores to detect nitrate toxicosis and congenital hypothyroidism in less sensitive animals, like affected foals that do not display any obvious clinical signs of goiter, but do display remarkable lesions of congenital musculoskeletal abnormalities.

On Thoroughbred horse farm B over the previous several foaling seasons, before the dietary changes were made to reduce nitrate in the diet, the foaling rates were between 10 to 40 percent each year. Most reproductive losses were caused by poor conception rates, early and late abortions, and congenital musculoskeletal abnormalities. Because of congenital defects during the previous foaling seasons, the mares delivering foals with anomalies were affected with dystocia and some mares succumbed because of foaling complications associated with malformed foals.

Our results and observations on the fetal loss syndrome on the two Thoroughbred horse farms in central Kentucky were shown to be associated with nitrate toxicosis. These findings are similar to fetal losses occurring on other farms. In the early 1980s, a newly emerging congenital musculoskeletal abnormality syndrome was first reported to occur in foals in western Canada [13-17], and later a case in eastern Canada (18). Recently, the syndrome has been reported in Europe
[19]. The syndrome is characterized by hyperplasia of the thyroid gland and concurrent musculoskeletal deformities. Congenital musculoskeletal defect syndrome is commonly reported as congenital hypothyroidism and dysmaturity syndrome (CHD), and is characterized by thyroid follicle hyperplasia, increased gestational length and multiple congenital musculoskeletal abnormalities, including mandibular prognathism [20, 21]. The thyroid gland epithelium is hyperplastic and the follicles are small, with minimal amounts of colloid. Affected foals have a thyroid gland that is grossly normal or only slightly increased in size (14).

Etiology of CHD is unclear, but dietary deficiencies of iodine, excess nitrate, and toxic substances have all been suggested [15-17]. Mares of affected foals appear to be unaffected $[13,20]$ and there does not appear to be a breed or sex predilection in foals affected with CHD, so a genetic link is unlikely (15). Thyroid hormone levels (T4 and T3) from CHD-affected foals can be low or normal, but the response to thyroid-stimulating hormone administration is invariably decreased [21].

eased [21]. Pregnant mares were fed immature cereal crops and did not receive any supplemental minerals, especially diets low in iodine, were more likely to produce a CHDaffected foal when compared with mares not exposed to these factors. The odds of one or more CHD foals being born on a farm feeding forage with a trace of nitrate were 8 times greater than the odds of the disease occurring on a farm that fed forage free of nitrate [17].

The CHD syndrome is unique and restricted primarily to western Canada, and northern Europe. Since the growing season is shorter in northern environments where the CHD syndrome is occurring, a common practice is to feed immature forages such as green-chop or haylage since climatic conditions are not conducive for processing dry hay. Immature forages are naturally high in nitrate. Other etiologic factors that need to be considered are toxic weeds that contaminate pasture forages. If forages are infested with toxic weeds, horses would be more likely to consume weeds that would be avoided if processed as conventional dry hay. Locoweeds are reported to cause limb abnormalities in foals [22]. Other toxic weeds, like buttercups or Ranunculus spp. are cool-season toxic plants endemic in western Canada and northern Europe where the CHD syndrome is occurring and they need to be investigated as they are abortigenic in mares [23]. Haylage or green-chop pasture forages are not fed to pregnant mares in central Kentucky because previously we associated these types of forages with multiple reproductive losses and deaths in adult horses.

It has been suggested that two different congenital defect syndromes appear to be occurring in foals [16]. We agree with these conclusions and feel that the CHD syndrome may be closely associated with the feeding of haylage and/or green-chop to pregnant mares, and another unique syndrome primarily associated with nitrate per se may be occurring in central Kentucky and elsewhere. This is the reason why it is imperative to improve the recognition of different syndromes 
that may be occurring [16]. The syndrome that occurs in central Kentucky $[8,9]$ and a similar syndrome reported in England [10] appear to be identical, but different than the CHD syndrome.

The reproductive loss syndrome, including limb contractures in foals, is common and widespread with a previously unknown etiology. This syndrome has been recognized in central Kentucky and elsewhere for several years [8-10]. The nitrogen fertilization of pastures appears to be a factor associated with acute spontaneous abortions in mares and other foals affected later in gestation with congenital thyroid hypothyroidism, and musculoskeletal abnormalities, primarily affecting the limbs of foals. Also, droughts, cold-stress and herbicide usage exacerbate nitrate accumulation in pastures in the spring.

In addition to the nitrogen fertilization of pastures, nitrogen is added to pasture forages by legumes like white clover. There is a natural spread of white clover in pastures following a prolonged drought that may reduce grass forages. When the drought is broken with adequate rainfall, volunteer white clover and weeds will aggressively grow, and the grass pastures become primarily white clover. When this climatic phenomenon occurs, it is referred to as "clover years", and there is a natural elevation of nitrate and protein in pastures in the spring. If there are unseasonable late spring frosts and freezes, nitrate will increase to toxic levels in pasture forages. Erratic climatic conditions with late frosts and freezes, and abundant white clover in pasture forages occurred in central Kentucky in 1980 and 2001 and massive, unprecedented reproductive losses occurred in mares and other herbivores during both foaling seasons.

Seemingly, developing fetuses of Boer goats are very sensitive to goitrogenic insults from nitrate. Paradoxically, the thyroid gland of fetuses from pregnant mares exposed to the same apparent goitrogenic agent as pregnant Boer goats from excessive nitrate and affected with reproductive losses do not display gross lesions of congenital thyroid hyperplasia as do kid goats, but reproductive losses and congenital musculoskeletal lesions are very apparent. Neither adult pregnant goats nor mares with affected offspring with congenital hypothyroidism display any clinical signs of goiter.

Nitrate competes with iodide uptake by the thyroid and has been associated with alterations in iodine metabolism, thyroid activity, and thyroid gland morphology in several animal species [24-26]. The obvious difference between species in how the thyroid gland responds morphologically to the apparently same goitrogenic agent is the reason why we did not associate hypothyroidism in foals with nitrate toxicosis and musculoskeletal abnormalities in earlier studies [9]. Were it not for the work of others [13-17] and the simultaneous observations and findings with pregnant Boer goats and pregnant mares consuming the similar high nitrate pasture forages, it would have been challenging to have made the association between nitrate, reproductive losses, congenital hypothyroidism and musculoskeletal abnormalities in foals that are less sensitive to the same goitrogenic agent as kid goats. However, the association was made possible by our unwavering clinical and pathologic findings over several years and observing the adverse effects of nature involving natural spontaneous cases in different species.

After our findings were suggested to other Thoroughbred horse breeding farms with reproductive losses, immediate improvements in reproductive foaling rates with fewer acute spontaneous abortions and the elimination of musculoskeletal abnormalities occurred on other horse farms in central Kentucky. Drastic erratic climatic changes cannot be controlled, but when spring pastures were not fertilized with nitrogenous fertilizers, herbicides not applied, protein reduced in the grain rations and sodium increased in the diet, reproductive losses were minimized [27].

Adequate sodium appears to alleviate excessive nitrate in the diet [3]. Cold-stressed plants react by increased levels of potassium and nitrate, and a decrease in sodium. It has been observed that cold-stressed, high nitrate pasture forages that induce grass tetany in cattle are also simultaneously associated with sodium deficiency, reproductive losses, including congenital musculoskeletal abnormalities in foals [3]. There is an association between hyponatremia and hypothyroidism (28). It has been demonstrated that coldstressed fescue grass resulted in a decrease in sodium in affected plants, and the decreased sodium in pasture forage may play an important role in the pathogenesis of grass tetany in cattle [29]. In addition, reproductive losses, neonatal diseases, including Tyzzer's disease in foals, are more common if diets high in nitrogenous compounds are fed to lactating mares [30].

The mysterious reproductive loss syndrome affected all herbivores during the increase of abortions in 2001 in central Kentucky [5]. Similarly, during the late 1950s and for many years earlier, increased abortions, with hundreds of fetal losses each spring in beef and dairy cattle were reported in Wisconsin and referred to as the Lowland abortion syndrome [31]. The cause was suspected to be and later associated with nitrate toxicosis. Many dairy cows are now losing pregnancies with an unknown etiology [32]. The clinical histories and the lack of significant laboratory findings in the Lowland abortion syndrome and current pregnancy losses in dairy and beef cows are strikingly similar to the MRLS. It is suspected that feeding of high nitrogenous diets and nitrate toxicosis may be a common etiologic factor with reproductive losses in mares, beef and dairy cows, goats, and other herbivores. We have observed similar pathologic lesions in poultry and swine fed high protein diets with fetal losses of unknown etiologies.

As with the Lowland abortion syndrome in beef and dairy cattle in Wisconsin, we also observed in central Kentucky in 2001 and after, early fetal losses are more common if mares were grazing pastures in low-lying areas near streams where the soil is higher in nitrogenous compounds [27]. Similarly, the EAFL in Australia occurs in the Hunter Valley in lowland areas. Lowland areas are more susceptible to frosts and freezes and more likely to be affected with fog and haze, 
which contain sulfur dioxide and nanoparticles [12], that may settle on pasture forages. In the spring of 2001 in central Kentucky the unseasonable climatic conditions of frosts and freezes also included marked fog and haze during the days preceding the onset of the massive, unprecedented fetal losses. We also observed that the fetal loss syndrome was more prevalent in low-lying areas that have high sulfur drinking water for pregnant mares.

A variety of sulfur sources can result in excessive sulfur intake, including water, feed ingredients and forages. Alfalfa, with its high protein and sulfur-containing amino acid content, is a significant source of sulfur. We have found that pregnant mares affected with the MRLS were prone to abortion if they were being fed high-protein alfalfa hay in addition to grazing pasture forages high in white clover that is also high in sulfur amino acids. Sulfonated minerals are also a source of sulfate in feed rations.

The rationale for lowering dietary protein and nitrogenous compounds is to offset the spike in protein and nitrate that naturally occurs in lush pastures in the spring. Adding nitrogenous fertilizers to lush pastures exacerbates the potential for additional nitrate accumulation. If nitrogenous fertilizers are applied to pastures, a less problematic time to apply them would be in the fall, rather than in the spring, when pastures are normally higher in nitrate and protein, and when pregnant mares and other herbivores are vulnerable for nitrate toxicosis.

It is difficult to demonstrate that nitrate toxicosis per se is responsible for fetal losses and congenital defects. However, since thyroid function and thyroid hormones are essential for early fetal development and the maintenance of pregnancy [33] and nitrate interferes with thyroid function, then the pathogenesis of nitrate toxicosis is also associated with reproductive losses and congenital defects. Nitrate levels at the time of abortions, or at birth may be normal, but earlier in fetal development, nitrate may be elevated to the extent it affects normal early thyroid function, which is essential for fetal development and for the prevention of reproductive losses, congenital defects, including musculoskeletal abnormalities.

The etiology of the reproductive loss syndrome is seemingly associated with nitrate toxicosis, but the pathologic lesions in the placenta of affected fetuses are suggestive of a non-contagious pathogenic entity, but none can be isolated. Our discovery of the nitrate, ammonium and sulfate-induced micro and nanoparticles explains the previously puzzling phenomenon as the pathogenic abiotic nanoparticles can mimic pathogenic microorganisms on how they multiply, and the lesions they produce. These nanoparticles are consistent findings in fetuses affected with the fetal loss syndrome. Seemingly, these pathogenic nanoparticles predispose fetuses to a multitude of opportunistic non-pathogenic microorganisms of different types, which may or may not be present, in addition to the toxic pathogenic nanoparticles that are consistently present in affected fetuses. Nitrate per se may not be that toxic, but ammonia as a by-product of excessive nitrate in combination with sulfate may be problematic for the production of toxic pathogenic nanoparticles. In addition to nitrate toxicosis, ammonia toxicosis, as a product of excessive nitrate, was a common finding and a suggested contributing etiologic factor for the MRLS [6].

Natural nanoparticles may be inorganic or organic matrixes of a mineral and a complex of amino acids, peptides, proteins, or other compounds that are 1 to $100 \mathrm{~nm}$ in diameter. Seemingly, the nanoparticles associated with the fetal loss syndrome are autogenous matrixes that are induced by toxicities to the components of amnionic fluid and plasma of developing fetuses.

Scanning electron microscopic examinations of affected tissues specimens revealed that the micro and nanoparticles originate as micro vesicles with dense centers that divide multiple times into two smaller vesicles approximately one half in size after each division. With each division the coccid vesicles with dense centers become smaller micro particles, then nanoparticles 1 to $100 \mathrm{~nm}$ in diameter. Nanoparticles are perceived as foreign bodies and because of their minute size can travel throughout the body inducing a chronic inflammatory response in many organs [34]. Often nocardiform placentitis in mares, that has an unknown pathogenesis, occurs concurrently with vascular lesions due to the pathogenic nanoparticles. It is hypothesized that nanoparticles induce niduses in the body of the uterus and/or placenta that predispose opportunistic microorganisms, including those associated with nocardiform placentitis, to proliferate and induce placentitis. The same appears to be occurring with a host of commensal and non-pathogenic opportunistic microorganisms [7].

Nanoparticles in fog and haze and environmental pollution are reported to be associated with deaths in animals and humans. With these studies, they hypothesized that the nitrate to nitric oxide pathway is involved with nitric oxide oxidizing sulfur dioxide to sulfate that is essential for the formation of nanoparticles. They suggest that nitrate in the environment may need to be decreased to reduce the formation of the ammonia and sulfate-induced nanoparticles [12].

Seemingly, potentially toxic nanoparticles may be ubiquitous in nature if environmental conditions are optimal for their formation. Nanoparticles were found in plants, especially those fertilized with nitrogenous compounds and infected with apparent fungal and bacterial opportunistic diseases [7]. The nanoparticles, like in animal diseases, may be predisposing plants to a host of opportunistic diseases. Nanoparticles were also found in honey bees affected with the colony collapse disorder. The affected honey bees were being supplemented with high-protein compounds and multiple sulfonated minerals similar to those that were pathogenic for early developing chicken embryos as described in section 4.5.

The discovery of nanoparticles of various types is rather new and nanotechnology is a rapidly emerging field involving many commercial applications. However, very little is known about any potential detrimental effects 
nanoparticles may have on human and animal health, and more research is needed to establish their safety [35].

The fetal loss syndrome is consistent with a toxicosis related entity that may affect all species of animals. The emergence of unexplained disease syndromes in invertebrates and vertebrates may be associated with drastic, erratic climate changes, but in addition, may be associated with changes in which foods and feedstuffs are currently formulated. In light of our findings regarding reproductive losses and secondary opportunistic diseases of animals, the adding of sulfur compounds, including sulfonated minerals to nitrogenous feedstuffs, and fermentation protein by-products that may be normally high in sulfur, may be exacerbating toxic nanoparticle formation in addition to that from environmental pollution. Sulfonated minerals have replaced the oxide and carbonate minerals during recent decades because they are more soluble and absorbable. Since the sulfonated minerals are more soluble and reactive, they are more likely to react with other compounds, including nitrogenous compounds and proteins in feedstuffs. Consequently, the sulfonated minerals may be problematic for the unintended consequence of forming toxic and pathogenic nanoparticles, but the oxide and carbonate minerals, which were previously more commonly used in feedstuffs, would not be as reactive and therefore are less likely to induce the toxic nanoparticles as we have demonstrated in our in vitro studies. Our findings that nitrate, ammonia and sulfate are associated with the formation of pathogenic nanoparticles confirm the recent findings by other international workers [12].

Since nanoparticles are routinely found in association with placental lesions and no other pathogenic microorganisms are present, the nanoparticles per se appear to be associated with the pathognomonic lesions in the amnion and amnionic umbilical cord, including malformations in the umbilical cord. Seemingly, the pathogenic abiotic nanoparticles provide niduses for secondary microorganisms associated with reproductive and opportunistic diseases of the respiratory and gastrointestinal tracts. In some fetuses, infarction in the amnion may be associated with nanoparticle-induce niduses in small vessels and in these cases fetal diarrhea is often present. Other than the pathogenic abiotic nanoparticles found in the intestinal contents of fetal diarrhea cases, no other significant pathogenic agents can be routinely demonstrated. In addition to placental lesions, pneumonic and intestinal lesions are common in late-term abortions.

In early developing embryos and fetuses, heart failure is the first clinical sign of impending early fetal death. Since the pathogenic abiotic nanoparticles have an affinity for the small vessels of the circulatory system, the vasculature of a developing heart is also affected. In adult horses on farms where the fetal loss syndrome is prevalent, we have found epicarditis and endopericarditis with massive edema formation in the pericardial sac. Other than presence of the pathogenic abiotic nanoparticles, no other significant microorganisms can be demonstrated in cases of epicarditis and endopericarditis in adult horses.
Our results show that pregnant Boer goats may be an ideal small animal model for the fetal losses associated with hypothyroidism and musculoskeletal abnormalities syndrome in large herbivores. In addition, fertile chicken eggs are ideal for the study of the reproductive loss syndrome in herbivores and other animals. We have found that a similar fetal loss syndrome with an unknown etiology is also occurring in poultry, goats, beef and dairy cows [32], and sows.

The fetal loss syndrome is complicated with a previous mysterious etiology. The syndrome is multi-factor and the nitrate-associated toxicosis can affect the fetus with multiple different conditions. If the thyroid is affected early in gestation and the fetus is not aborted, congenital musculoskeletal abnormalities may develop due to hypothyroidism. In the same or other fetuses, nitrate, ammonium and sulfate toxicosis may induce the formation of toxic pathogenic nanoparticles that produce amnionic, umbilical cord and circulatory lesions in various organs including the heart. The pathogenic nanoparticles act as foreign bodies due to their minute size and may travel throughout the body producing niduses in the small vessels for a host of secondary opportunistic microorganisms to proliferate [34].

The composition of the toxic pathogenic nanoparticles that form in the amnionic fluid and in pathologic lesions has yet to be determined. Their formation in amnionic fluid and plasma may be due to unintended consequences of saltinduced precipitation of autogenous amnionic and plasma proteins by toxic levels of ammonium sulfate and other sulfated electrolytes in the diet of affected pregnant animals.

\section{Conclusions}

Congenital hypothyroidism, musculoskeletal abnormalities and fetal losses in mares and other animals are seemingly associated with the effects of nitrate toxicosis. Abiotic pathogenic micro and nanoparticles associated with excessive nitrate, ammonia and sulfate are consistent findings in pathognomonic placental lesions with fetal losses of previously unknown etiology associated with the MRLS. The discovery of toxic pathogenic abiotic micro and nanoparticles in developing fetuses appears to be unique and a novel mechanism of action for the pathogenesis of fetal losses. Seemingly, the nanoparticles form niduses in small vessels and predispose animals to a host of opportunistic disease syndromes affecting the reproductive, respiratory and gastrointestinal tracts of affected animals. The potentially toxic nanoparticles may be ubiquitous in nature if environmental conditions are optimal for their formation. Toxic nanoparticles may be associated with the pathogenesis of opportunistic diseases of unknown etiologies in plants, animals and beneficial insects like honey bees.

\section{Acknowledgements}

The authors express gratitude to the owner of the Thoroughbred Stud Farm (AS) for allowing our unwavering 
suggestions to reduce nitrate in forages, fertilizers, herbicides, and dietary supplements for pregnant mares. We are also grateful that the farm submitted for pathological examination an average of 175 placentas each year, since 2003.

Our appreciation is extended to the veterinary practitioners in central Kentucky who provided fetuses and placentas from mares and other livestock since the 1980 breeding season for pathologic examination.

The views expressed in this paper are solely those of the authors. Dr. Alan Dorton is a private veterinary equine practitioner on call for the Department of Veterinary Science Equine Research Farm.

\section{Conflicts of Interest}

The authors declare there were no conflicts of interest.

\section{References}

[1] Swerczek, T. W. (1986) Equine Fetal Diseases. In Current Therapy in Theriogenology 2. DA Marrow editor. W. B. Saunders Company. 699-704.

[2] Swerczek, T. W. (1980) Early Fetal Death and Infectious Placental Disease in the Mare. Proc 26th Ann Conv AAEP. Anaheim, CA. 173-179.

[3] Swerczek T. W. (2018) Sodium for the Prevention of Grass Tetany and Fetal Losses Associated with Nitrate Toxicosis in Herbivores. Nutri Food Sci Int J. 8(1): 555728. DOI:10.19080/NFSIJ.2018.08.5.

[4] Cohen, N. D., Carey, V. J., Donahue, J. G., Seahorn, J. L, Donahue, J. K., D. M. Williams, D. M., Harrison, L. R. (2003) Case-control study of late-term abortions associated with mare reproductive loss syndrome in central Kentucky. J Am Vet Assoc, 222, 199-209.

[5] Taylor, J. R. (2002) Theory of ammonia toxicity as the mechanism of abortion in the mare reproductive loss syndrome. J Equine Vet Sci, 22, 237-239.

[6] Swerczek, T. W. (2007) Bacterial Diseases of the Fetus and Placenta Associated with Fetal Loss in the Mare: Early and Late Fetal Loss Syndrome. Current Therapy in Large Animal Theriogenology, 2 ed, Saunders Elsevier. St. Louis, Missouri. pp. 186-188.

[7] Swerczek, T. W. (2002) Saprotrophic Fungi and Bacteria and Commensal Bacteria that infect Frost Damaged Pastures may be Contributing to Gut Microbial Overgrowth and Lesions Associated with the Mare Reproductive Loss Syndrome. J. of Equine Veterinary Science, 22, 234-237.

[8] Rooney, J. R. (1966) Contracted Foals. Cornell Vet, 56, 172-187.

[9] Crowe, M. W., Swerczek, T. W. (1985) Equine Congenital Defects. AJVR, 46, 355-358.

[10] Wilsher, S., Ousey, J., Allen, W. R. (2013) Observations on the placentae of eight Thoroughbred foals born with flexural limb deformities. Equine Veterinary Education, 25, 84-95.

[11] Perkins, N. R., Todhunter, K. H., Wylie, R. M., A. E. Begg, A.
E., Gilkerson, J. R. Muscatello, G., Racklyeft, D. J., Chicken, C., Wilson, M. C. (2006) Equine amnionitis and foetal loss (EAFL): a newly recognized condition in Australia. Proceedings of the 11th Symposium of the International Society for Veterinary Epidemiology and Economics, Cairns, Australia, Aug. p. 934.

[12] Wang, G., Zhang, R., Gomez, M. E., Yang, L. (2016) Persistent Sulfate formation from London Fog to Chinese haze. Proc Natl Acad Sci USA, 113, 13630-13635.

[13] McLaughlin, B. G. (1981) Hyperplastic goiter in newborn foals in western Canada. Can Vet J, 22, 42-45.

[14] McLaughlin, B. G., Doige, C. E. (1981) Congenital musculoskeletal lesions and hyperplastic goiter in foals. Can Vet J, 22, 130-133.

[15] Kreplin, C., Allen, A. L. (1991) Congenital hypothyroidism in foals in Alberta. Can Vet J, 32, 751.

[16] Allen, A. L., Doige, C. E., Fretz, P. B., Townsend, H. G. G. (1994) Hyperplasia of the thyroid gland and concurrent musculoskeletal deformities in western Canadian foals: reexamination of a previously described syndrome. Can Vet J, $35,31-38$.

[17] Allen, A. L, Townsend, H. G. G., Doige, C. E., Fretz, P. B. (1996) A case-control study of the congenital hypothyroidism and dysmaturity syndrome of foals. Can Vet J, 37, 349-358.

[18] Gawrylash, S. K. (2004) Thyroid hyperplasia and musculoskeletal deformity in a Standardbred filly in Ontario. Can Vet J, 45, 424-426.

[19] Koikkalainen, K., Knuuttila, A., Karikoski, N., Syrjä, P., Hewetson, M. (2014) Congenital hypothyroidism and dysmaturity syndrome in foals: First reported cases in Europe. Equine Veterinary Education, 26, 181-189.

[20] Allen, A. L. (1995) Hyperplasia of the thyroid gland and musculoskeletal deformities in two equine abortuses. Can Vet J, 36, 234-236.

[21] McLaughlin, B. G., Doige, C. E., McLaughlin, P. S. (1986) Thyroid hormone levels in foals with congenital musculoskeletal lesions. Can Vet J, 27, 264-267.

[22] Mcllwraith, C. W., James, L. F. (1982) Limb deformities in foals associated with ingestion of locoweed by mares. J Am Vet Med Assoc, 181, 255-258.

[23] Swerczek, T. W. (2016) Abortions in Thoroughbred mares associated with consumption of bulbous buttercups (Ranunculus bulbosus L). JAVMA, 248, 669-672.

[24] Bloomfield, R. A, Welsch, C. W., Garner, G. B., M. E. Muhrer, M. E. (1961) Effect of dietary nitrate on thyroid function. Science, 134, 1690.

[25] Chaoui, A. A., Zaki, A, Talibi, A. Chait, A. Derouche, T. Aboussaouira, F. Benabdjlil, T. Himmi. (2004) Effects of inorganic nitrates on thyroid gland activity and morphology in female rats Therapie, 59, 471-475.

[26] Eskiocak, S., Dundar, C., Basoglu, T., Altaner, S. (2005) The effects of taking chronic nitrate by drinking water on thyroid functions and morphology. Clin Exp Med, 5, 71.

[27] Swerczek, T. W, Dorton, A. R. (2001-2018) Evidence-based field trial results and observations on Thoroughbred horse breeding farms in central Kentucky. Unpublished data. 
[28] Hanna, F., Scanlon, M. F. (1997) Hyponatraemia, hypothyroidism, and role of arginine-vasopressin. The Lancet, $350,755-756$.

[29] Blevins, D. G., Remley, M., Lukaszewski, K. Davis, D. (2011) The loss of sodium in freeze damaged tall fescue forage could be a major contributor to spring grass tetany. Forage and Grazinglands, Online, doi: 10.1094/FG.

[30] Swerczek, T. W. (2013) Tyzzer's Disease in Foals: Retrospective studies from 1969 to 2010. Canadian Veterinary Journal, 54, 876-880.

[31] Simon, J., Sund, J. M., Wright, M. J., Winter, A., Douglas, F. D. (1958) Pathological Changes Associated with the Lowland Abortion Syndrome in Wisconsin. 132, 164-169.
[32] Britt, J., Alvarez, F. (2011) Why are so many cows losing pregnancies? Losing up to 20 percent of pregnancies is not acceptable. Hoard's Dairyman, 156, 751-752.

[33] Maruo, T., Katayama, K., Matuso, H. Anwar, M., Mochizuki, M. (1992). The role of maternal thyroid hormones in maintaining early pregnancy in threatened abortion Acta Endocrinologica, 127, 118-122.

[34] Gatti, A., Montanari, S. (2008) Nanopathology: The health impact of nanoparticles. Pan Stanford Publishing, Pte. Ltd Singapore, ISBN -10981\-4241-00-8, pp. 1-298.

[35] McClements, D. J., Xlio, H. (2017) Is nano safe in foods? Establishing the factors impacting the gastrointestinal fate and toxicity of organic and inorganic food-grade nanoparticles. npj Science of Food, Article number 6. 